\title{
Neural Network-Based Coronary Heart Disease Risk Prediction Using Feature Correlation Analysis
}

\author{
Jae Kwon Kim and Sanggil Kang \\ Department of Computer Engineering, Inha University, Incheon, Republic of Korea \\ Correspondence should be addressed to Sanggil Kang; sgkang@inha.ac.kr
}

Received 10 April 2017; Revised 5 July 2017; Accepted 12 July 2017; Published 6 September 2017

Academic Editor: Eddie Ng Yin Kwee

Copyright (c) 2017 Jae Kwon Kim and Sanggil Kang. This is an open access article distributed under the Creative Commons Attribution License, which permits unrestricted use, distribution, and reproduction in any medium, provided the original work is properly cited.

\begin{abstract}
Background. Of the machine learning techniques used in predicting coronary heart disease (CHD), neural network (NN) is popularly used to improve performance accuracy. Objective. Even though NN-based systems provide meaningful results based on clinical experiments, medical experts are not satisfied with their predictive performances because $\mathrm{NN}$ is trained in a "black-box" style. Method. We sought to devise an NN-based prediction of CHD risk using feature correlation analysis (NN-FCA) using two stages. First, the feature selection stage, which makes features acceding to the importance in predicting CHD risk, is ranked, and second, the feature correlation analysis stage, during which one learns about the existence of correlations between feature relations and the data of each NN predictor output, is determined. Result. Of the 4146 individuals in the Korean dataset evaluated, 3031 had low CHD risk and 1115 had CHD high risk. The area under the receiver operating characteristic (ROC) curve of the proposed model $(0.749 \pm 0.010)$ was larger than the Framingham risk score (FRS) $(0.393 \pm 0.010)$. Conclusions. The proposed NN-FCA, which utilizes feature correlation analysis, was found to be better than FRS in terms of CHD risk prediction. Furthermore, the proposed model resulted in a larger ROC curve and more accurate predictions of CHD risk in the Korean population than the FRS.
\end{abstract}

\section{Introduction}

According to the World Health Organization (WHO), coronary heart disease (CHD) is one of the most dangerous diseases in the world. According to the WHO, around 17.7 million people died from CHD in 2015 [1]. CHD includes hyperlipidemia, myocardial infarction, and angina pectoris [2-4]. In general, medical experts arrive at diagnoses based on electrocardiography, sonography, angiography, and blood test results. CHD is not easily diagnosed during the early disease stage [5-8], but for effective treatment, its early diagnosis is important [9]. However, diagnoses are made based on medical experts' personal experiences and understanding of the disease, which increase the risks of errors, delay appropriate treatment, increase treatment times, and substantially increase costs. In order to solve these problems, many studies have been conducted on clinical decision support systems
[10] using various techniques, such as data mining and machine learning [11-15]. Of the machine learning techniques that have been used to predict $\mathrm{CHD}$, neural network $(\mathrm{NN})$ is popularly used to improve performance accuracy $[9,16-20]$. NN is good at generalizing data without domain knowledge of CHD prior to training. In addition, by analyzing complex data, $\mathrm{NN}$ makes it possible to discover new patterns and information related to $\mathrm{CHD}$ [21-23].

Although the NN-based systems mentioned above have provided meaningful results based on clinical experiments, medical experts remain dissatisfied with $\mathrm{NN}$, because of its "black-box" characteristics [24-26], that is, predictors are trained without knowledge of relationships between input features and NN outputs. Many CHD-related features are used to train CHD predictors. Unnecessary or unimportant features for predicting $\mathrm{CHD}$ can be included during predic- 


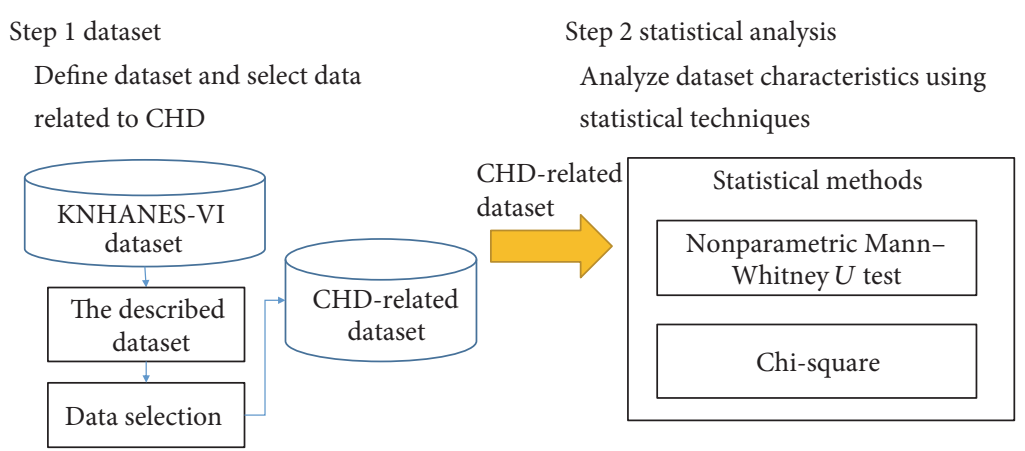

Step 5 performance measure Measure the PPV, NPV, and accuracy of the proposed method

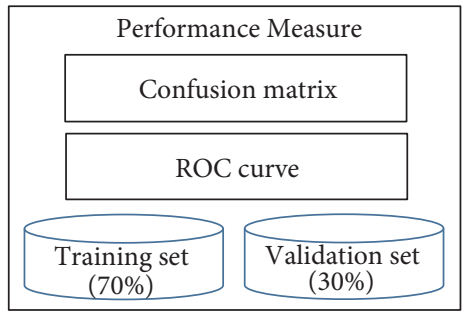

Propose prediction model

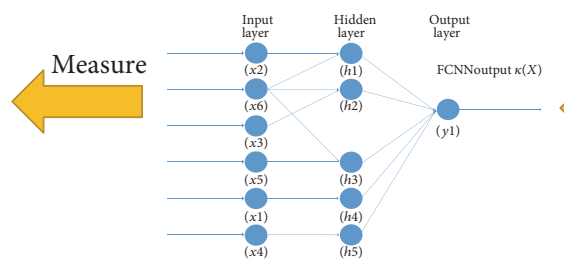

NN-based CHD prediction model using feature correlation analysis

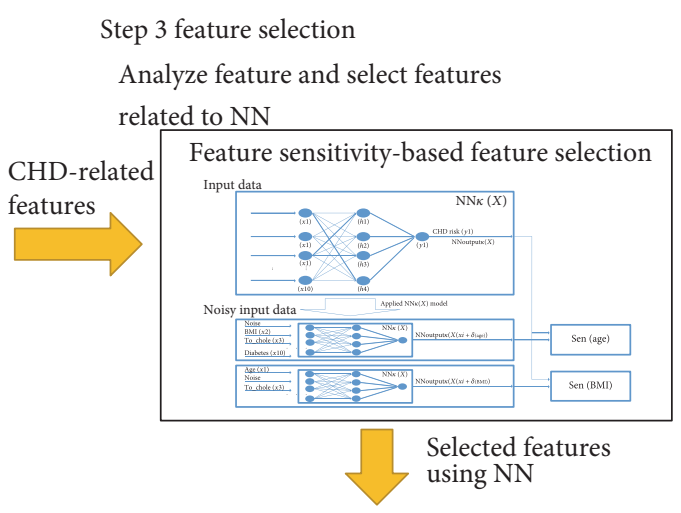

Step 4 partial correlation analysis Analyze correlation of mutually sensitive features for NN configuration

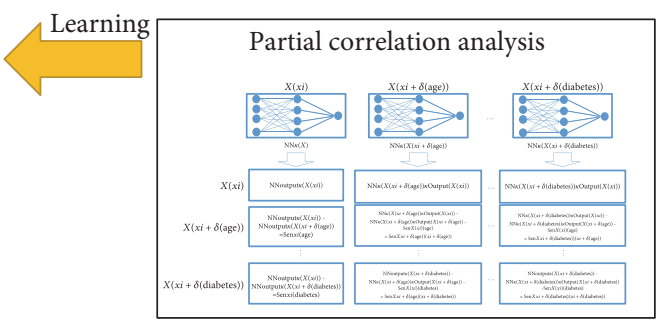

FIgURe 1: Study design.

tor training. In this case, when the new data is input, it does not predict correctly.

In this paper, we propose an NN-based CHD risk prediction method based on feature correlation analysis (NNFCA), which includes two processes, that is, feature selection and feature correlation analysis.

(i) First, during the feature selection stage, we ranked features with respect to their importance for predicting CHD risk. Rankings were calculated using feature sensitivity in a trained NN. Based on these rankings, $\mathrm{NN}$ was retrained after eliminating the lowest ranked features in a stepwise manner. This process was continued until the performance of the $\mathrm{NN}$ degraded as compared with the previous stage. Once necessary features were obtained using this process, we analyze the NN to know relationship between the features in generating NN output in order to model an NN predictor which can avoid the black-box style training.

(ii) Second, during the feature correlation analysis stage, we analyzed features to identify feature relations and determine whether they were correlated with $\mathrm{NN}$ predictor outputs. If features were affected on contribution to predictor output by changing in any of them, features were considered correlated. The NNbased CHD predictor using feature correlation analysis is trained in the way that correlated features are connected in coupled and uncorrelated features are decoupled.
To prove the predictive accuracy of our method, we used the 6th Korea National Health and Nutrition Examination Survey (KNHANES-VI) dataset [27] and evaluated the performances between Framingham risk scores (FRSs) $[28,29]$, other machine learning techniques, and proposed NN-FCA.

The remainder of this paper consists of the following: Chapter 2 describes the proposed method; Chapter 3 detains results; Chapter 4 provides a discussion; and finally, our conclusions are stated in Chapter 5.

\section{Method}

The study design is shown in Figure 1. During step 1, KNHANES-VI dataset was examined and data was selected. In step 2, statistical analysis was performed to identify features related to CHD risk. In step 3, predictors of $\mathrm{CHD}$ risk were selected using feature sensitivity-based feature selection. In step 4, NN-based CHD risk predictors were trained using feature correlation analysis of features. In step 5, performance measurements were made to validate NN-based CHD risk predictions using feature correlation analysis.

2.1. Dataset. The KNHANES-VI was conducted by the Korea Centers for Disease Control and Prevention. KNHANES identifies the health and nutritional status of the population that provides the statistics required to assess whether health policies are being effectively delivered. It also provides statistical data on smoking, drinking, physical 
activity, obesity, and disease requested by the World Health Organization (WHO) and the Organization for Economic Cooperation and Development (OECD) [27].

We use the KNHANES-VI dataset to perform CHD risk prediction. Input variables for training were age, sex, body mass index (BMI), total cholesterol (To_chole), HDL cholesterol, systolic blood pressure (SBP), diastolic blood pressure (DBP), triglyceride, hemoglobin, thyroid disease (TD), chronic renal failure (CRF), hepatitis type B (H_B), hepatitis type C (H_C), cirrhosis, smoking, and diabetes. The output variables used were $\mathrm{CHD}$ riskrelated variables, that is, hypertension, dyslipidemia, stroke, myocardial infarction, and angina. When these five diseases are not present and do not exist, CHD is of low risk, but if one of the five is present, $\mathrm{CHD}$ is of high risk. 8108 record set of KNHANES-VI was used for the experiment. We excluded 3324 uncertain (nonrespondent, "Null" value) respondents and 638 records of individuals under 30 years old. The final CHD-related dataset comprised 4146 records.

2.2. Statistical Analysis. The nonparametric Mann-Whitney $U$ test (continuous features) and the chi-square (categorical features) were used to compare age, sex, BMI, To_chole, HDL, SBP, DBP, triglyceride, hemoglobin, TD, CRF, H_B, H_C, cirrhosis, smoking, and diabetes in the low- and highrisk groups. The statistical analysis was performed using IBM SPSS Ver. 22.0 [30]. Several preoperative features were compared to determine the most effective method of CHD risk prediction.

Confusion matrix and receiver operating characteristics (ROC) curve [31] were used for performance comparison. Confusion matrix provides a means of evaluating the performance of the classifier as shown in Table 1 [32]: positive predictive value (PPV), negative predictive value (NPV), and accuracy (1). PPV and NPV are the proportions of positive and negative results with true positive or true negative results, respectively. PPV and NPV describe the performance of diagnostic tests or other statistical measures [33]. The accuracy of a measurement system is the degree of closeness of measurements of a quantity to that quantity's true value [34]. It is constructed for output variable (CHD low risk, $\mathrm{CHD}$ high risk) in the validation dataset of each analysis. The limit of significance for all tests is $P<0.05$.

$$
\begin{aligned}
\mathrm{PPV} & =\frac{\mathrm{TP}}{\mathrm{TP}+\mathrm{FP}} \\
\mathrm{NPV} & =\frac{\mathrm{TN}}{\mathrm{TN}+\mathrm{FN}} \\
\text { Accuracy } & =\frac{\mathrm{TP}+\mathrm{TN}}{\mathrm{TP}+\mathrm{FP}+\mathrm{FN}+\mathrm{TN}} .
\end{aligned}
$$

2.3. Feature Selection. From $n$ features extracted for classifying low and high risks, we select features based on importance in contribution to good classification. The importance of each feature is measured by feature sensitivity from a trained NN predictor. The ith feature sensitivity, denoted as
TABLE 1: Confusion matrix.

\begin{tabular}{cccc}
\hline \multirow{2}{*}{ Confusion matrix } & \multicolumn{2}{c}{ Prediction } \\
& Positive & Negative \\
\hline \multirow{2}{*}{ Actual } & True positive (TP) & False positive (FP) \\
& Negative & False negative (FN) & True negative (TN) \\
\hline
\end{tabular}

$\operatorname{Sen}\left(X, x_{i}\right)$, is calculated by an average of $\mathrm{NN}$ output changes between original dataset and noisy dataset which is generated by adding a very small noise (denoted as $\delta$ ) to $x_{i}$. The $i$ th feature sensitivity is

$$
\operatorname{Sen}\left(X, x_{i}\right)=\frac{1}{N} \sum_{\forall_{k}} \mid \text { NNoutput }_{k}\left(X_{\left(x_{i}+\delta\right)}\right)-\text { NNoutput }_{k}(X) \mid,
$$

where $\operatorname{NNoutput}_{k}(X)$ and NNoutput ${ }_{k}\left(X_{(x i+\delta)}\right)$ are the outputs for the input, $k$, with an original input dataset, $X$, and the output with a noisy input $\left(X_{(x i+\delta)}\right)$ obtained by adding a very small amount of noise $\delta$ to the $i$ th feature, respectively. All feature sensitivities were calculated individually with one feature sensitivity. The $\delta$ value was generated randomly within the range [a1, 0.0010]. Figure 2 presents a schematic diagram of the methodology for calculating the feature sensitivity using NN. All feature sensitivities were sorted in a descending order, and the feature with the lowest sensitivity of the feature set was eliminated. The $\mathrm{NN}$ was retrained using the remaining features and then verified to determine whether the performance is not degraded compared to that of the original $\mathrm{NN}$ trained using all features. If the performance is not degraded, then the aforementioned process repeats until the necessary features are determined.

2.4. Feature Correlation Analysis. To overcome the performance limitation of $\mathrm{NN}$ due to the characteristics of black-box training [24-26], prior information on the correlation relationship among the features was acquired using the feature sensitivity change in generating NNoutputs. The correlated features are connected to the hidden layer in a coupled connection. On the other hand, the uncorrelated features are connected in uncoupled connection. The sensitivity of a feature in a trained $N N$ means the relative importance index in generating NNoutput. This contains the intention that if the magnitude of a feature increases, the importance of the feature increases while training NN. Moreover, if the magnitude of the increase in the feature affects the other features significantly, the corresponding features can be considered to be correlated with each other. To determine if the features are correlated or uncorrelated, this study examined the changes in feature sensitivity, as seen in the algorithm in Pseudocode 1. Figure 3 gives an example of the NN prediction model trained based on the feature relations, such as correlated and uncorrelated. 


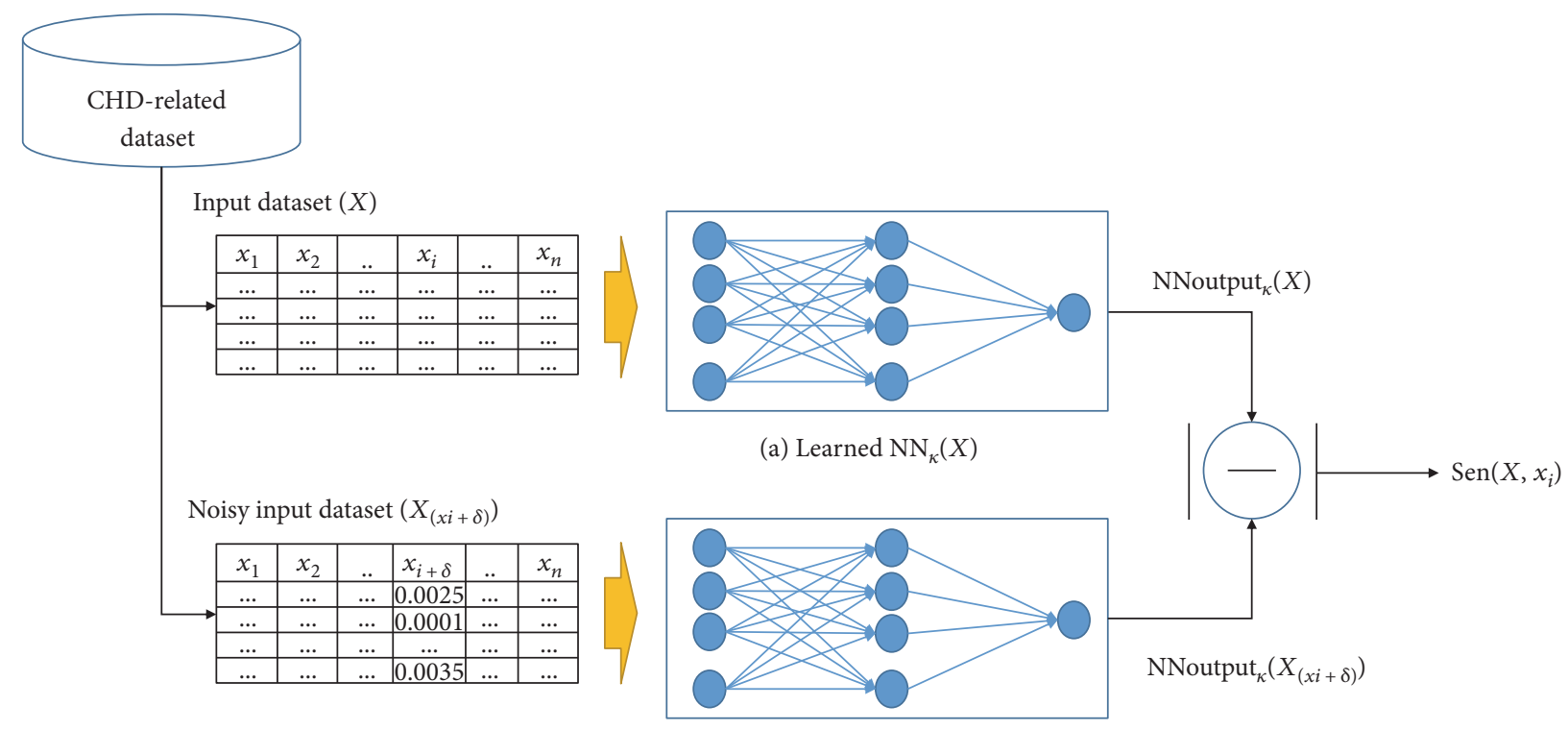

(b) The learned $\mathrm{NN}_{\kappa}(X)$ same as (a)

Figure 2: A schematic diagram of calculating the feature sensitivity using NN.

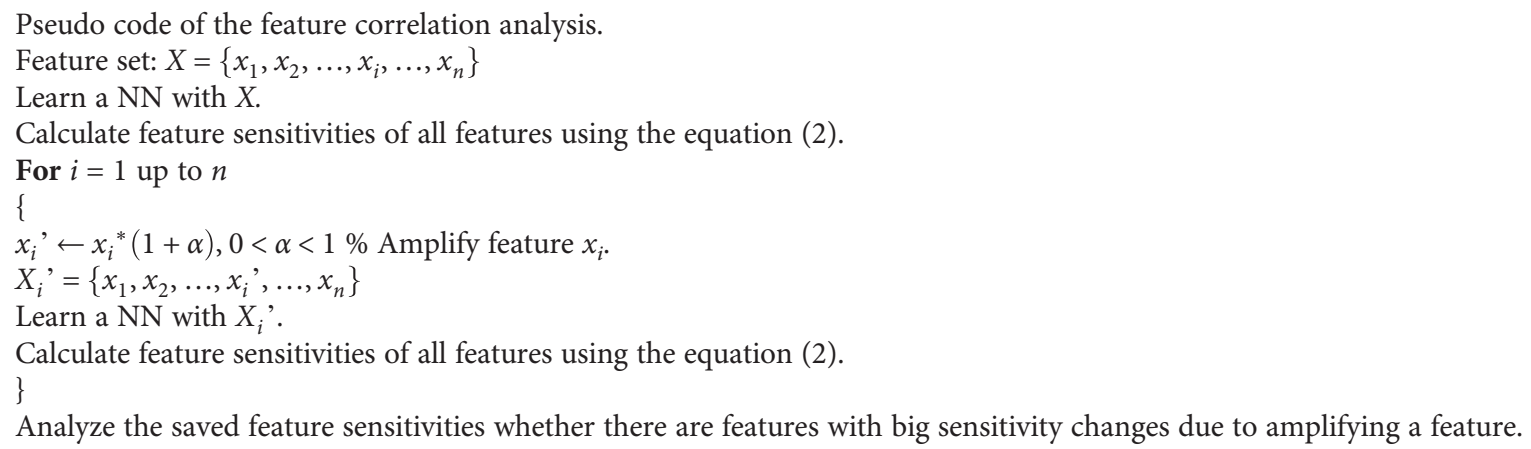

Pseudocode 1

\section{Result}

3.1. Characteristics. Table 2 lists the distribution of the preoperative parameters between the people at low risk and high risk of CHD.

The median age of the 4146 subjects was 52 years (range: 30-92; mean: 52.501). The median low-risk age and high-risk age were 47 years (range: 30-87; mean: 48.60 ) and 64 years (range: 30-92; mean: 63.11), respectively. The median BMI was 23.68 (range: 15.302-41.304; mean: 23.969). The median low-risk BMI and high-risk BMI were 23 (range: 15-40; mean: 23.594) and 25 (range: 16-41; mean: 25.004), respectively. The median To_chole level was $189 \mathrm{mg}$ (range: 79525; mean: 190.974). The median low-risk To_chole level and high-risk To_chole level were $190 \mathrm{mg}$ (range: 89-384; mean: 191.738) and $185 \mathrm{mg}$ (range: 79-525; mean: 188.898), respectively. The median HDL was $50 \mathrm{mg}$ (range: 22-118; mean: 51.843). The median low-risk HDL and high-risk HDL were $51 \mathrm{mg}$ (range: 22-111; mean: 52.642) and $48 \mathrm{mg}$ (range: $23-118$; mean: 49.671), respectively. The median SBP level was $117 \mathrm{mmHg}$ (range: 75-219; mean: 118.979). The median low-risk SBP level and high-risk SBP level were $113 \mathrm{mmHg}$ (range: 75-209; mean: 155.583) and $127 \mathrm{mmHg}$ (range: 88-219; mean: 128.209), respectively. The median DBP was $75 \mathrm{mmHg}$ (range: 10-137; mean: 75.822). The median low-risk DBP level and high-risk DBP level were $75 \mathrm{mmHg}$ (range: 44-137; mean: 75.61) and $76 \mathrm{mmHg}$ (range: 10-118; mean: 76.397), respectively. The median triglyceride level was $112.5 \mathrm{mmol} / \mathrm{L}$ (range: 201868; mean: 139.236). The median low-risk triglyceride level 

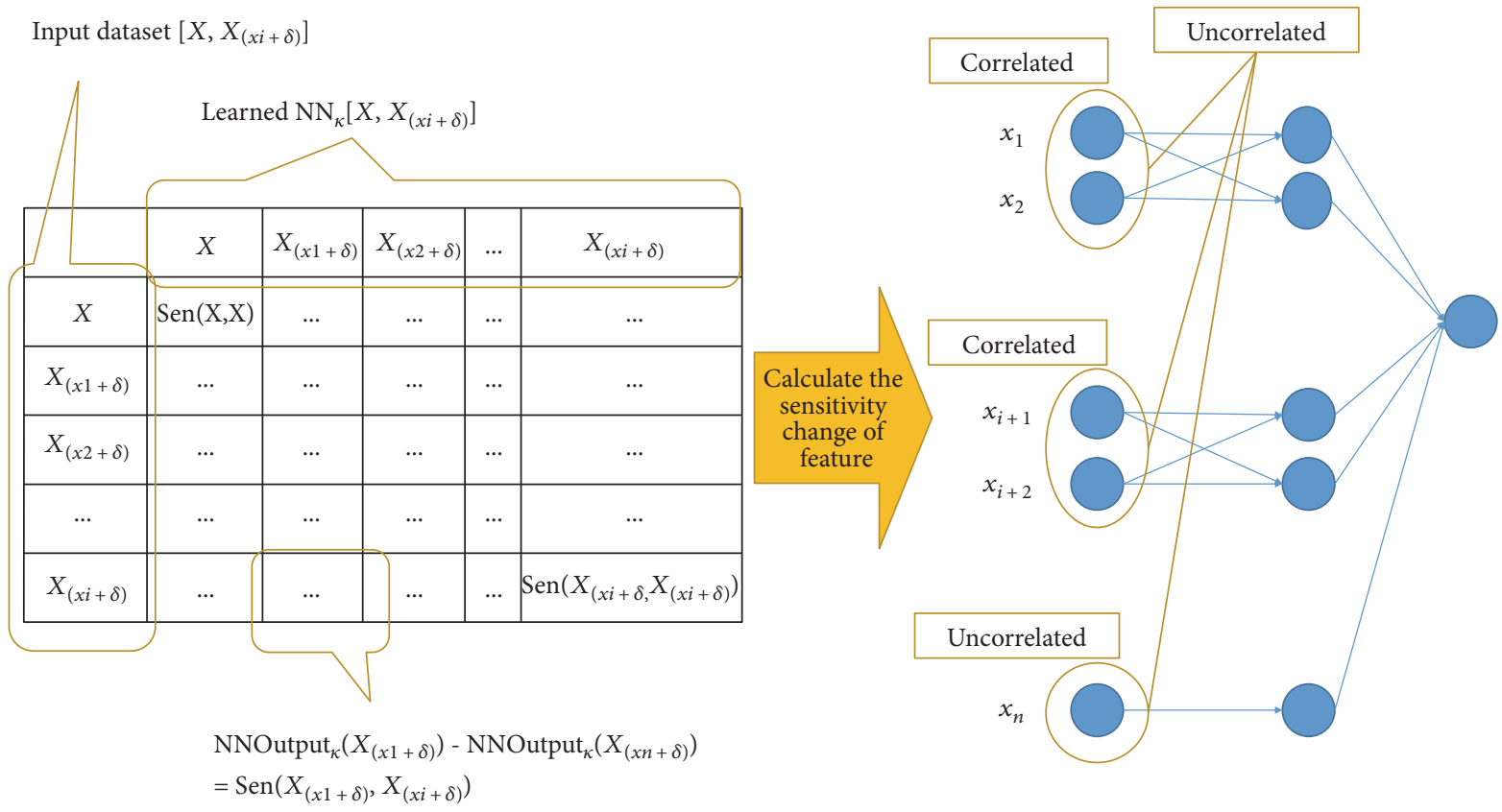

Figure 3: An example of NN predictor using the feature correlation analysis.

and high-risk triglyceride level were $106 \mathrm{mmol} / \mathrm{L}$ (range: 201868; mean: 131.570) and $129 \mathrm{mmol} / \mathrm{L}$ (range: 28-1397; mean: 160.0744), respectively. The median hemoglobin level was $13.9 \mathrm{mg} / \mathrm{dl}$ (range: 6.7-19.1; mean: 13.981). The median low-risk hemoglobin level and high-risk hemoglobin level were $14 \mathrm{mg} / \mathrm{dl}$ (range: 7-19; mean: 14.057) and $14 \mathrm{mg} / \mathrm{dl}$ (range: 7-18; mean: 13.989), respectively. The difference between the 2 groups (low risk and high risk) in age, BMI, To_chole, HDL, SBP, DBP, and triglyceride was significant (independent $t$-test): $P=0.001$ (age), $P=0.001$ (BMI), $P=0.024$ (To_chole), $P=0.001$ (HDL), $P=0.001$ (SBP), $P=0.035$ (DBP), $P=0.001$ (triglyceride), and $P=$ 0.206 (hemoglobin). The 4146 subjects were classified according to sex as female (1777) and male (2369). The TD was classified as no (4073) and yes (73). The CRF was classified as no (4134) and yes (12). The H_B was classified as no (4117) and yes (29). The H_C was classified as no (4143) and yes (3). Cirrhosis was classified as no (4136) and yes (10). Smoking was classified as no (3322) and yes (824). Diabetes was classified as no (2625). An impaired fasting glucose was classified as no (994) and yes (527). The difference between the 2 groups (low risk and high risk) in sex, TD, CRF, H_B, H_C, cirrhosis, smoking, and diabetes triglyceride was significant (chi-square test): $P=0.893$ (sex), $P=0.370$ (TD), $P=$ 0.022 (CRF), $P=0.933$ (H_B), $P=0.801$ (H_C), $P=0.349$ (cirrhosis), $P=0.001$ (smoking), and $P=0.001$ (diabetes).

3.2. Feature Sensitivity-Based Feature Selection Result. $\mathrm{NN}_{k}(X)$ consisted of 16 input nodes, 4 hidden nodes, and one output node. Noisy data $\left(x_{i}-\delta\right)$ were applied to the trained $\mathrm{NN}_{k}(X)$ to calculate the sensitivity of each feature. Figure 4 outlines the calculation process of the feature sensitivity.
Table 3 presents the results of the feature sensitivity. From the Table, To_chole (0.100), age (0.081), SBP (0.073), and DBP (0.049) are considered the important features for $\mathrm{CHD}$ risk predictor. The $\mathrm{NN}$ is retrained by removing the lowest ranked feature one at a time until the performance of the NN degrades, as shown in Table 4. The best performance was obtained when only seven features (sex, hemoglobin, TD, CRF, H_B, H_C, and cirrhosis) were removed, with an $81.163 \%$ accuracy of predicting CHD.

3.3. NN-Based CHD Risk Predictor Using Feature Correlation Analysis. From the result in Section 3.2, the nine features (age, BMI, To_chole, HDL, SBP, DBP, triglyceride, smoking, and diabetes) were selected and used for feature correlation analysis, as shown in Figure 5 . The correlated features of each feature were determined according to the mutual effects on the sensitivity changes. In other words, the correlated features influenced their sensitivity changes in one another due to the amplification of a single feature. For example, the change in feature sensitivity of SBP was 0.017 when it was amplified, which is denoted as $X\left(_{\mathrm{SBP}+\delta}\right)$, as listed in Table 5. The amplification on SBP is believed to have been affected by the sensitivity changes of three features, such as BMI (0.025), To_chole (0.042), and DBP (0.017), because they showed much or higher sensitivity changes than the average sensitivity change $(0.017)$ of all the features. To verify the mutuality of the correlation, the sensitivity change of SBP was analyzed according to the amplification on BMI, To_chole, and DBP, respectively. For the amplification on BMI $\left(X\left(_{\mathrm{SBP}+\delta}\right)\right)$, the sensitivity change of SBP is 0.007 , which is much less than the average sensitivity 
TABLE 2: Characteristics (continuous variable: mean; categorical variable: count).

\begin{tabular}{|c|c|c|c|}
\hline Feature & $\begin{array}{c}\text { Low risk } \\
\text { (3031 people) }\end{array}$ & $\begin{array}{c}\text { High risk } \\
\text { (1115 people) }\end{array}$ & $P$ value \\
\hline Age & 48.600 & 63.110 & 0.326 \\
\hline Sex & & & 0.893 \\
\hline Male & 1301 & 476 & \\
\hline Female & 1739 & 639 & \\
\hline BMI & 23.594 & 25.004 & 0.001 \\
\hline To_chole & 191.738 & 188.898 & 0.024 \\
\hline HDL & 52.642 & 49.671 & 0.001 \\
\hline SBP & 115.583 & 128.210 & 0.001 \\
\hline DBP & 75.610 & 76.397 & 0.035 \\
\hline Triglyceride & 131.570 & 160.074 & 0.001 \\
\hline Hemoglobin & 14.057 & 13.989 & 0.206 \\
\hline TD & & & 0.370 \\
\hline No & 2981 & 1092 & \\
\hline Yes & 50 & 23 & \\
\hline CRF & & & 0.002 \\
\hline No & 3027 & 1092 & \\
\hline Yes & 4 & 23 & \\
\hline H_B & & & 0.933 \\
\hline No & 3010 & 1107 & \\
\hline Yes & 21 & 8 & \\
\hline H_C & & & 0.801 \\
\hline No & 3029 & 1114 & \\
\hline Yes & 2 & 1 & \\
\hline Cirrhosis & & & 0.349 \\
\hline No & 3025 & 1111 & \\
\hline Yes & 6 & 4 & \\
\hline Smoking & & & 0.001 \\
\hline No & 2350 & 972 & \\
\hline Yes & 681 & 143 & \\
\hline Diabetes & & & 0.001 \\
\hline No & 2167 & 458 & \\
\hline Impaired fasting glucose & 671 & 323 & \\
\hline Diabetes & 193 & 334 & \\
\hline
\end{tabular}

change (0.012) of all features. Therefore, BMI is not considered to be correlated with SBP. For the amplification on To_chole $\left(X\left(_{\mathrm{To} \_ \text {chole }+\delta}\right)\right)$, SBP was not correlated, similar to the BMI. On the other hand, for the amplification of $\mathrm{DBP}\left(X\left(_{\mathrm{DBP}+\delta}\right)\right)$, the sensitivity change of SBP was 0.035 , which is larger than the average sensitivity change (0.022) of all features. Overall, the analysis showed that the SBP and DBP are correlated with each other. The correlated features for the remaining features were examined in the same way. Based on the correlation of features, the NN-based CHD risk predictor, in which the correlated features are coupled in connection to the hidden layer, was modelled, as seen in Figure 6 .
For example, BMI and DBP were coupled in connection to the hidden layer because both are correlated with each other.

3.4. Performance Measure. The performance of the proposed $\mathrm{NN}$-based CHD risk prediction was examined using feature correlation analysis, and the results were compared with those obtained by feature correlation analysis (NN_FCA) with logistic regression (LR), neural network (NN), and Framingham risk score (FRS) [28], using the performance metrics, such as confusion matrix (positive predictive value (PPV), negative predictive value (NPV), and accuracy) and ROC curve. The experimental dataset was divided into training set (70\%) and validation set (30\%). Table 6 lists the results of the performance measure.

From Table 5, FRS showed a lower performance with an accuracy of $28.87 \%$. LR and NN gave high performance (80.32\% and $81.09 \%$, resp.), but the performance was lower than that of NN_FCA. NN_FCA showed the best performance compared to the other models in both the training set and validation set $(87.63 \%$ and $82.51 \%)$. The PPV and NPV also showed the highest NN_FCA (71.29\% and $85.70 \%$, resp.) than the other models. The accuracy of NN_FCA was highest at $82.51 \%$ because the correlation relationship of the features is trained while training NN_FCA.

The results of the ROC curve are shown in Table 7 and Figure 7. As shown on the left of the figure, FRS has a very low ROC area of $0.393 \pm 0.010$. Because FRS is a statistical method suitable for a specific population and environment, it appears to be unfit for the Korean population. LR and NN were $0.713 \pm 0.010$ and $0.735 \pm 0.010$, respectively. Here, $\mathrm{NN}$ was found to be effective for predicting the CHD risk, as reported in a previous study $[17,35]$. On the other hand, as shown on the right of the figure, NN_FCA was $0.749 \pm 0.010$, which was better than the existing $\mathrm{NN}$, because it removes the unnecessary features when training the prediction model. In other words, the sensitivity-based feature selection can effectively detect the features associated with a prediction of the risk of CHD.

As a result, the error rate can be reduced using NN_FCA because it removes the unnecessary connections between the nodes in NN. Therefore, NN_FCA is excellent in terms of the performance accuracy. The proposed NN_FCA is effective for predicting the risk of $\mathrm{CHD}$.

\section{Discussion}

$\mathrm{NN}$ is a training method that imitates the human brain and is a very successful technique for predicting the relationship between the input values and target values. In addition, it is a predictive model for supporting a back propagation method and a powerful technique that can help in determining the support involved in the problems of classification, inference, prediction, and sequential reasoning [36, 37]. Substantial research has attempted to predict the CHD risk; LR and $\mathrm{NN}$ are used typically in machine learning. The prediction performance degrades because unnecessary features are considered during training LR and NN $[9,16-20]$. The 


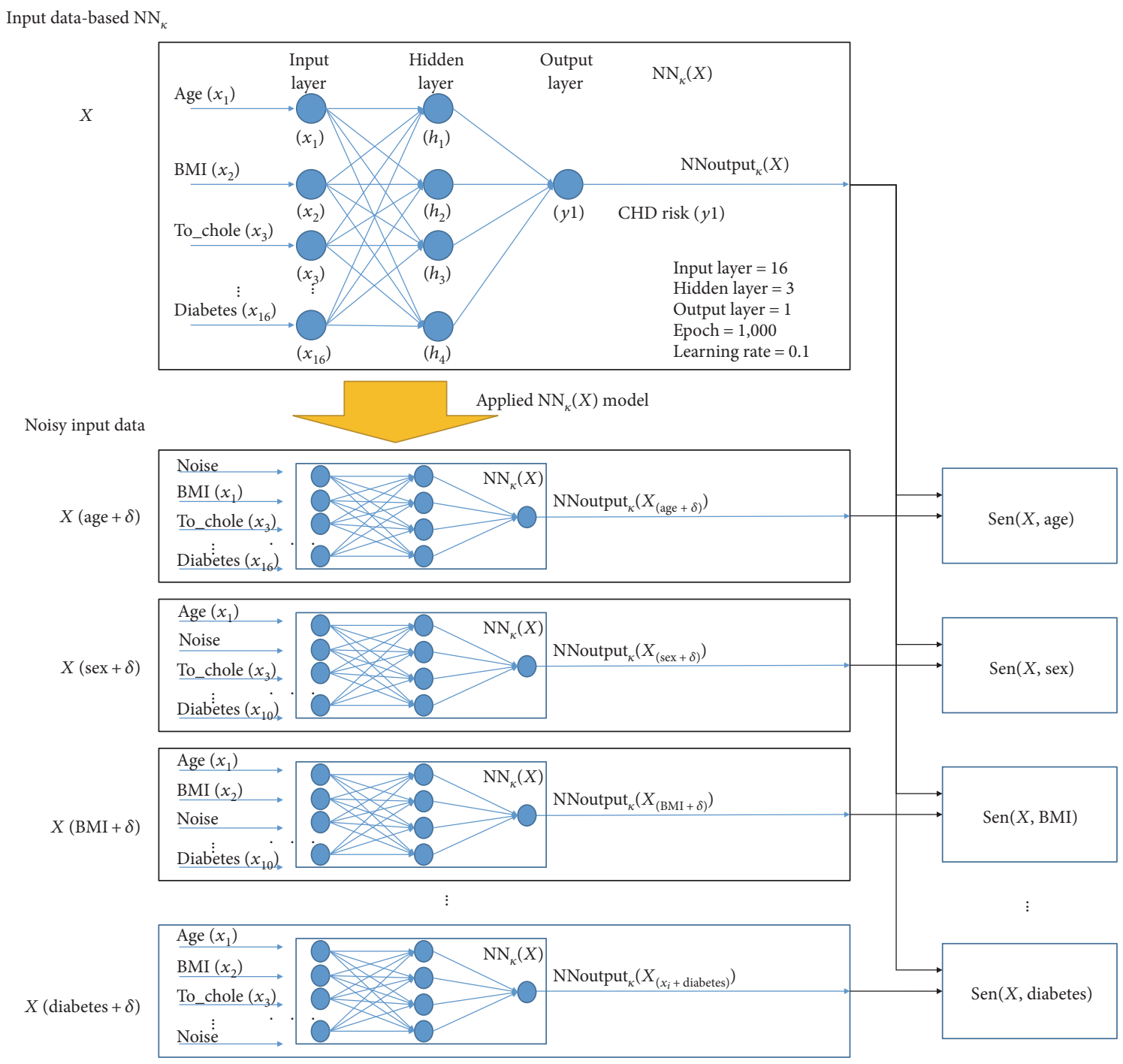

FIGURE 4: Calculation process of the feature sensitivity.

proposed method solves this problem by removing the unnecessary features using sensitivity-based feature selection.

The most popular decision support of the risk of CHD is the Framingham risk score (FRS) [28], which provides the $\mathrm{CHD}$ risk index with a statistical technique using the patients' demographics and various medical examination information. Currently, the accuracy of the FRS is $28.87 \%$, as evaluated using the KNHANES-VI dataset [27]. The FRS has difficulty in reflecting the environments, which change with time, and is limited to patients in a specific region because it uses the U.S. patients' data collected from 1960 to 1970 [29].

Many studies have been conducted to predict the risk of CHD using machine learning. Arabasadi et al. [35] proposed a hybrid neural network genetic for a $\mathrm{CHD}$ risk prediction in 2017. In this work, the input features were selected using a genetic algorithm and the CHD predictor was then modelled with a neural network. Narain et al. [9] developed a CHD risk prediction system modelled with the quantum neural network in 2016. This work increased the quantum interval according to the error value of the output layer during training and provided weights to the sigmoid function. Verma et al. [16] proposed a novel hybrid method, in which feature selection, particle swarm optimization, and $\mathrm{K}$-means were used for a CHD prediction in 2016. They finally employed supervised learning, such as NN, LR, and fuzzy unordered rule induction as well as a C4.5 decision tree for classification. Zhao and $\mathrm{Ma}$ [17] proposed an intelligent noninvasive diagnosis system based on empirical mode decompositionTeager energy operator to estimate the instantaneous frequency of diastolic murmurs and back propagation NN to classify the murmurs in 2008 . They worked on classifying a normal group and CHD group according to the electrocardiogram (ECG) signal for diastolic murmurs. Akay [18] proposed a CHD predictor modelled using a NN in 1992. They presented a clinical demonstration from the data of 100 
TABLE 3: Results of feature sensitivity analysis.

\begin{tabular}{|c|c|c|c|}
\hline Features & Sensitivity & NNoutput-NNoutput $(x i+\delta)$ & Rank \\
\hline$\overline{\mathrm{NN}_{k}(X)}$ & 0.815 & & \\
\hline $\mathrm{NN}_{k}\left(X_{(x i-\text { age })}\right)$ & 0.734 & 0.081 & 2 \\
\hline $\mathrm{NN}_{k}\left(X_{(x i-\operatorname{sex})}\right)$ & 0.726 & 0.008 & 11 \\
\hline $\mathrm{NN}_{k}\left(X_{(x i-\mathrm{BMI})}\right)$ & 0.769 & 0.038 & 5 \\
\hline $\mathrm{NN}_{k}\left(X_{(x i-\text { To_chole })}\right)$ & 0.677 & 0.100 & 1 \\
\hline $\mathrm{NN}_{k}\left(X_{(x i-\mathrm{HDL})}\right)$ & 0.703 & 0.013 & 8 \\
\hline $\mathrm{NN}_{k}\left(X_{(x i-S B P)}\right)$ & 0.729 & 0.073 & 3 \\
\hline $\mathrm{NN}_{k}\left(X_{(x i-\mathrm{DBP})}\right)$ & 0.693 & 0.049 & 4 \\
\hline $\mathrm{NN}_{k}\left(X_{(x i \text {-triglyceride })}\right)$ & 0.753 & 0.013 & 7 \\
\hline $\mathrm{NN}_{k}\left(X_{(x i \text {-hemoglobin })}\right)$ & 0.796 & 0.006 & 12 \\
\hline $\mathrm{NN}_{k}\left(X_{(x i-\mathrm{TD})}\right)$ & 0.806 & 0.003 & 13 \\
\hline $\mathrm{NN}_{k}\left(X_{(x i-\mathrm{CRF})}\right)$ & 0.802 & 0.010 & 10 \\
\hline $\mathrm{NN}_{k}\left(X_{\left(x i-\mathrm{H} \_\mathrm{B}\right)}\right)$ & 0.813 & 0.001 & 15 \\
\hline $\mathrm{NN}_{k}\left(X_{\left(x i-\mathrm{H}_{-} \mathrm{C}\right)}\right)$ & 0.813 & 0.001 & 16 \\
\hline $\mathrm{NN}_{k}\left(X_{(x i-\text { cirrhosis })}\right)$ & 0.812 & 0.002 & 14 \\
\hline $\mathrm{NN}_{k}\left(X_{(x i-\text { smoking })}\right)$ & 0.802 & 0.012 & 9 \\
\hline $\mathrm{NN}_{k}\left(X_{(x i-\text { diabetes })}\right)$ & 0.786 & 0.024 & 6 \\
\hline
\end{tabular}

TABLE 4: Results of NNs eliminating the lowest ranked features (\%).

\begin{tabular}{lr}
\hline Without features & Accuracy \\
\hline Without 1 (H_C) feature & 77.743 \\
Without 2 (H_C and H_B) features & 78.518 \\
Without 3 (without 2 features and cirrhosis) features & 80.644 \\
Without 4 (without 3 features and TD) features & 80.920 \\
Without 5 (without 4 features and hemoglobin) features & 81.120 \\
Without 6 (without 5 features and sex) features & 81.141 \\
Without 7 (without 6 features and CRF) feature & 81.163 \\
Without 8 (without 7 features and smoking) features & 81.018 \\
Without 9 (without 8 features and HDL) features & 80.921 \\
Without 10 (without 9 features and triglyceride) features & 80.222 \\
Without 11 (without 10 features and diabetes) features & 79.522 \\
Without 12 (without 11 features and BMI) features & 79.209 \\
\hline
\end{tabular}

patients. Kukar et al. [19] proposed a CHD prediction system using the ECG data and modelled it with a Bayesian NN. Detrano et al. [20] developed a CHD prediction system modelled from the data of 425 patients using the LR technique. As mentioned above, CHD prediction studies using NNs are ongoing.

This study was conducted to predict the risk of CHD in Koreans. In general, heart disease is influenced by age, sex, BMI, total cholesterol, HDL, systolic blood pressure, diastolic blood pressure, smoking, and diabetes [38-46]. In Koreans, CHD was not found to be associated with sex, hemoglobin, thyroid disease, H_B, H_C, or cirrhosis disease
$(P$ value $<0.05)$. On the other hand, triglyceride and CRF were associated with $\mathrm{CHD}(P$ value $=0.035)$. Triglyceride is an important factor in predicting the risk of CHD. This study confirmed that triglyceride is a very important factor for $\mathrm{CHD}$ in Koreans. In addition, the results of NN-based $\mathrm{CHD}$ risk prediction using feature correlation analysis showed that SBP and DBP are correlated. This is reasonable because both have similar characteristics. In addition, BMI and DBP are closely related, that is, obese people have high blood pressure in general [47]. In addition, the relationship between DBP and total cholesterol affects CHD [48]. The proposed NN-based $\mathrm{CHD}$ risk prediction using feature 


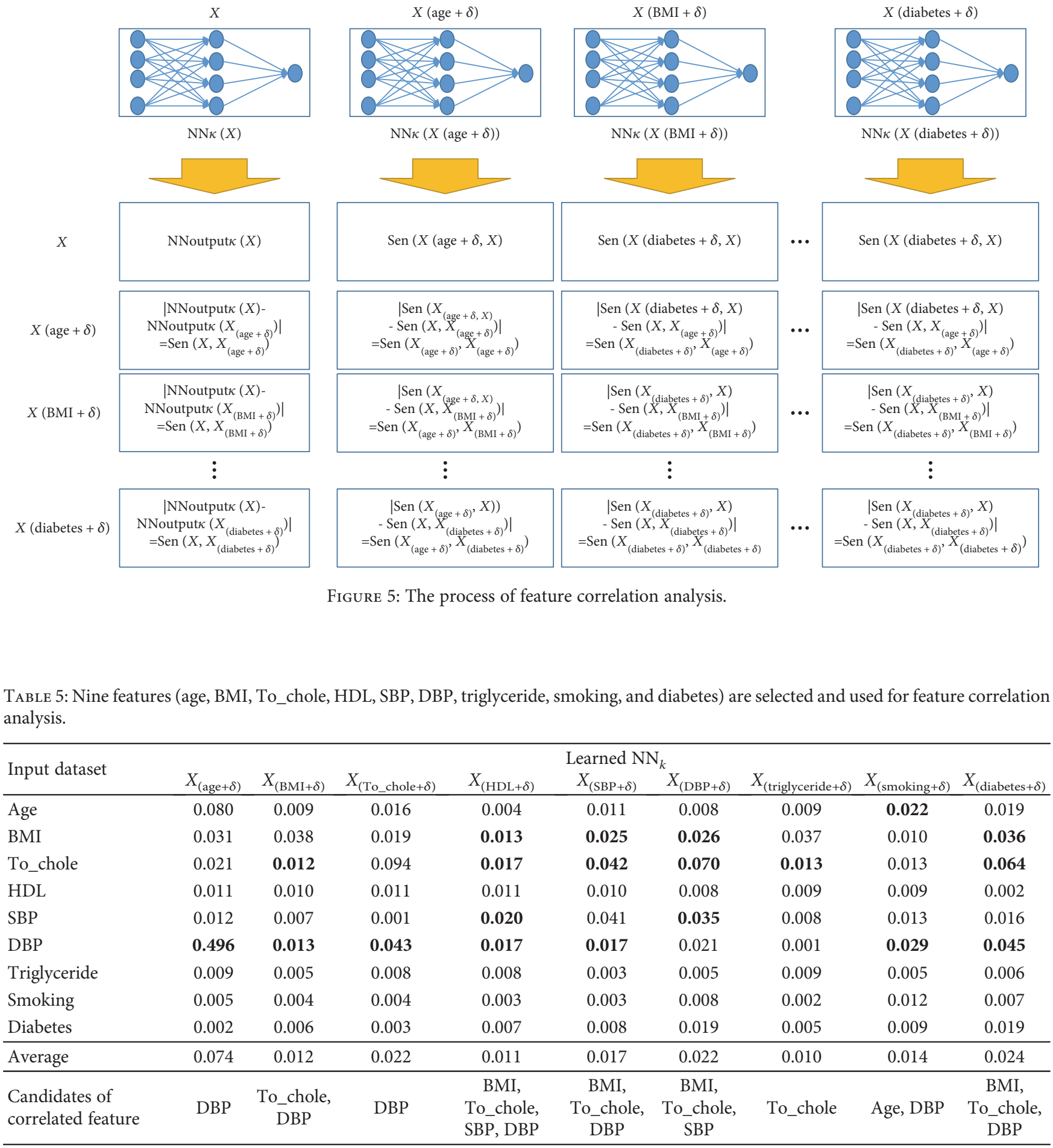

correlation analysis showed higher accuracy $(82.51 \%)$ in a $\mathrm{CHD}$ prediction compared to the other models and proved to be more useful than the FRS applied in the past.

\section{Conclusion}

This paper proposed an NN-based CHD risk prediction using feature correlation analysis (NN-FCA) and experimented with the KNHANES-VI dataset. The proposed model will improve the CHD risk and decision support for suitable treatment. Sex, hemoglobin, thyroid disease, H_B, H_C, and cirrhosis were not associated, whereas triglyceride and CRF were closely related to CHD. In addition, triglyceride is a very important factor in the risk of CHD in Koreans. Furthermore, the correlated features are BMI and DBP, DBP and total cholesterol, and SBP and DBP. The proposed model 
Correlation feature list:

(1) BMI, DBP

(2) DBP, To_chole

(3) DBP, SBP
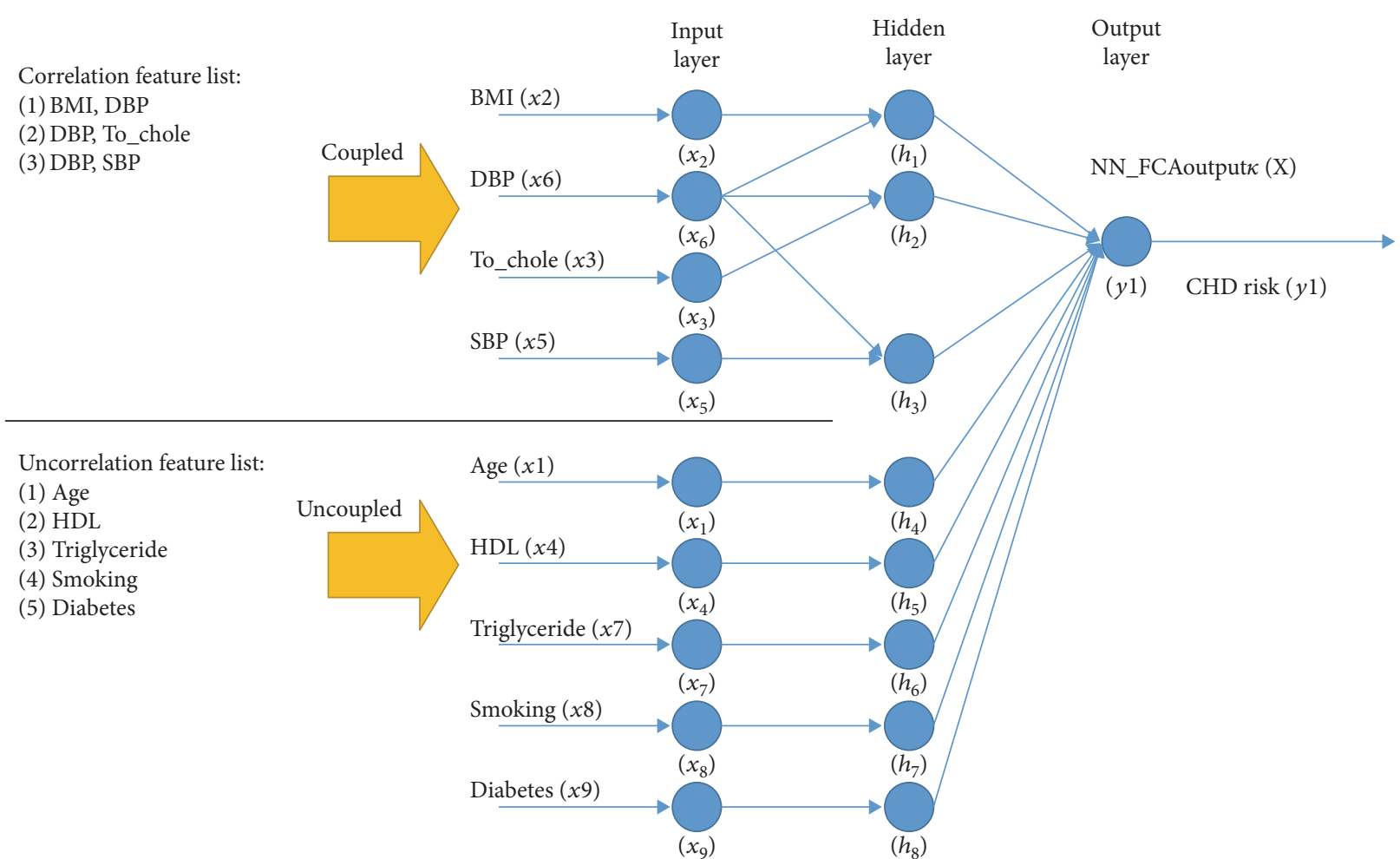

FIGURE 6: NN-based CHD prediction using feature correlation analysis.

TABLE 6: Results of performance measure with training set (\%).

\begin{tabular}{|c|c|c|c|c|c|c|}
\hline & \multicolumn{3}{|c|}{ Training set } & \multicolumn{3}{|c|}{ Validation set } \\
\hline & PPV & NPV & Accuracy & PPV & NPV & Accuracy \\
\hline LR & 57.24 & 87.63 & 86.11 & 67.53 & 83.63 & 80.32 \\
\hline $\mathrm{NN}$ & 63.04 & 88.67 & 87.04 & 67.55 & 85.08 & 81.09 \\
\hline FRS & 2.54 & 85.48 & 6.67 & 21.49 & 54.41 & 28.87 \\
\hline NN_FCA & 67.57 & 89.00 & 87.63 & 71.29 & 85.70 & 82.51 \\
\hline
\end{tabular}

TABLE 7: Results of ROC curve using validation set.

\begin{tabular}{lcccc}
\hline & & & \multicolumn{2}{c}{$95 \%$ Confidence Interval } \\
Upper bound \\
\hline LR & ROC curve & $P$ value & Lower bound & 0.732 \\
NN & $0.713 \pm 0.010$ & 0.001 & 0.693 & 0.754 \\
FSNN & $0.735 \pm 0.010$ & 0.001 & 0.716 & 0.760 \\
FRS & $0.741 \pm 0.010$ & 0.001 & 0.722 & 0.414 \\
NN_FCA & $0.393 \pm 0.010$ & 0.001 & 0.373 & 0.768 \\
\hline
\end{tabular}

was as good as FRS in terms of the CHD risk prediction. Compared to the validation of the FRS for the Korean population, the proposed model resulted in a larger ROC curve and more accurate $\mathrm{CHD}$ risk prediction.
The proposed model acknowledging such characteristics was developed, which may aid in the prevention of heart disease in these individuals. This might deliver great benefit to people in terms of predicting, beyond a simple prediction 


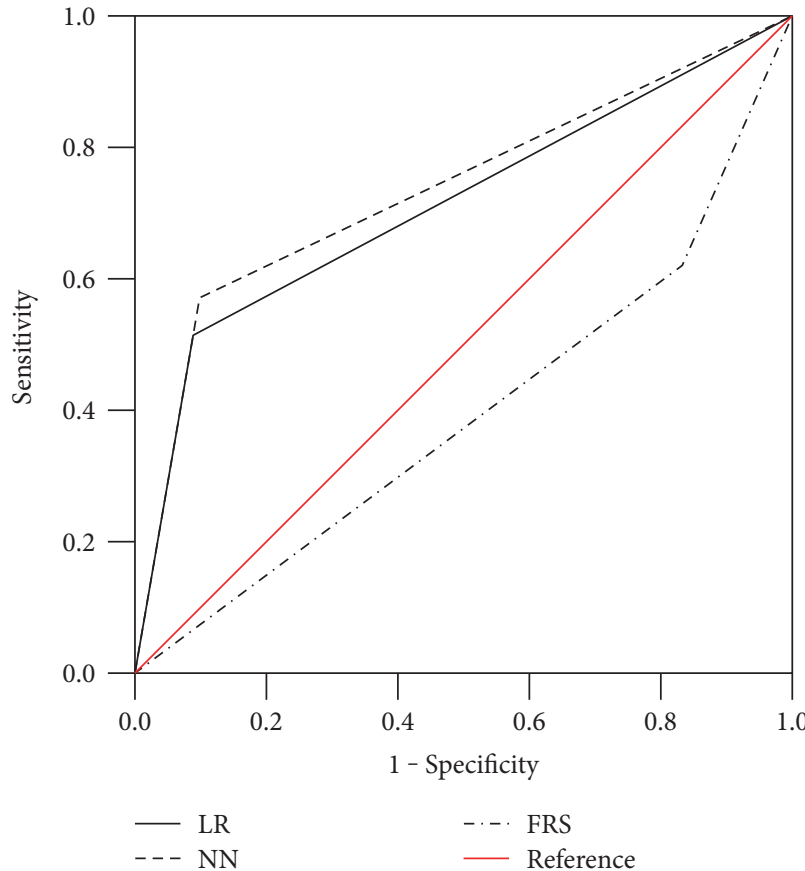

(a)

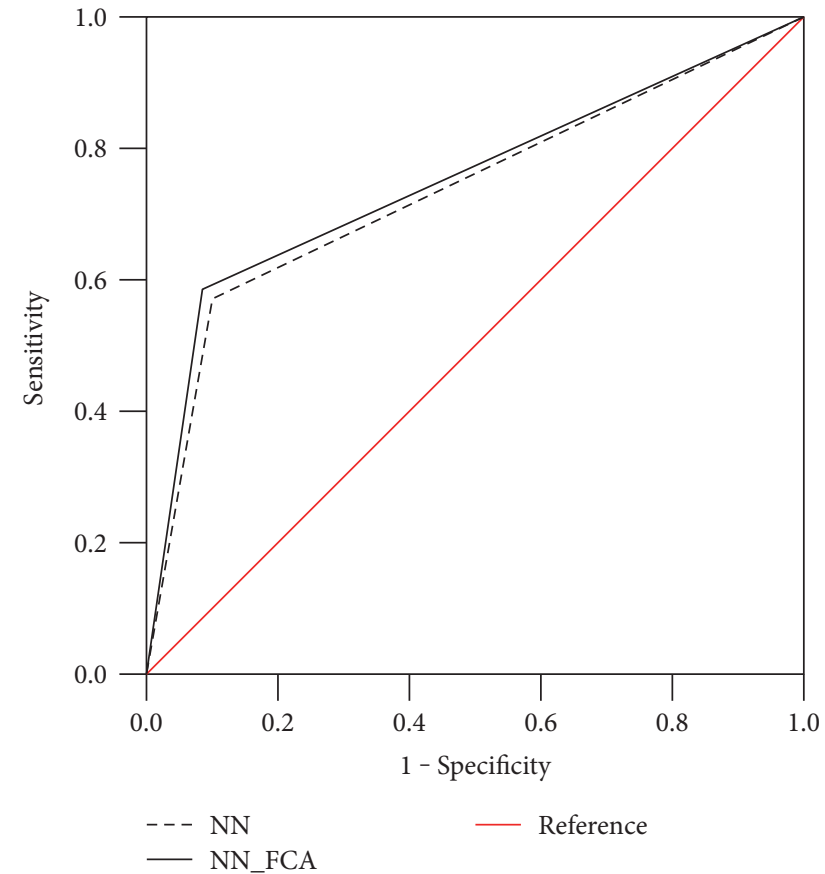

(b)

Figure 7: Result of ROC curve (a) compared to LR, NN, and FRS; (b) compared to NN and NN_FCA.

of the CHD risk and the quantitative survival time. Furthermore, a self-diagnosis algorithm or a similar clinical decision support system could be developed and applied meaningfully if the NN-FCA can be applied to diseases other than CHD.

\section{Conflicts of Interest}

The authors declare that they have no conflicts of interest.

\section{Acknowledgments}

This work was supported by Inha University research grant.

\section{References}

[1] Cardiovascular Diseases (CVDs) Fact Sheet N $N^{\circ} 17$, WHO, 2015, [updated May 2017]. http://www.who.int/mediacentre/ factsheets/fs317/en/index.html.

[2] Y. Maneerat, K. Prasongsukarn, S. Benjathummarak, W. Dechkhajorn, and U. Chaisri, "Intersected genes in hyperlipidemia and coronary bypass patients: feasible biomarkers for coronary heart disease," Atherosclerosis, vol. 252, pp. e183e184, 2016.

[3] T. Nakashima, T. Noguchi, S. Haruta et al., "Prognostic impact of spontaneous coronary artery dissection in young female patients with acute myocardial infarction: a report from the angina pectoris-myocardial infarction multicenter investigators in Japan," International Journal of Cardiology, vol. 207, pp. 341-348, 2016.

[4] J. S. Zebrack, J. L. Anderson, C. A. Maycock et al., "Usefulness of high-sensitivity C-reactive protein in predicting long-term risk of death or acute myocardial infarction in patients with unstable or stable angina pectoris or acute myocardial infarction," The American Journal of Cardiology, vol. 89, no. 2, pp. 145-149, 2002.

[5] W. B. Kannel, T. Gordon, W. P. Castelli, and J. R. Margolis, "Electrocardiographic left ventricular hypertrophy and risk of coronary heart disease. The Framingham study," Annals of Internal Medicine, vol. 72, no. 6, pp. 813-822, 1970.

[6] S. Cook, E. Ladich, G. Nakazawa et al., "Correlation of intravascular ultrasound findings with histopathological analysis of thrombus aspirates in patients with very late drug-eluting stent thrombosis," Circulation, vol. 120, no. 5, pp. 391-399, 2009.

[7] S. E. Nissen, E. M. Tuzcu, P. Libby et al., "Effect of antihypertensive agents on cardiovascular events in patients with coronary disease and normal blood pressure: the CAMELOT study: a randomized controlled trial," JAMA, vol. 292, no. 18 , pp. 2217-2225, 2004.

[8] R. O. Bonow, B. A. Carabello, K. Chatterjee et al., "2008 focused update incorporated into the ACC/AHA 2006 guidelines for the management of patients with valvular heart disease: a report of the American College of Cardiology/ American Heart Association Task Force on Practice Guidelines (writing committee to revise the 1998 guidelines for the management of patients with valvular heart disease) endorsed by the Society of Cardiovascular Anesthesiologists, Society for Cardiovascular Angiography and Interventions, and Society of Thoracic Surgeons," Journal of the American College of Cardiology, vol. 52, no. 13, pp. e1-e142, 2008.

[9] R. Narain, S. Saxena, and A. K. Goyal, "Cardiovascular risk prediction: a comparative study of Framingham and quantum neural network based approach," Patient Preference and Adherence, vol. 10, pp. 1259-1270, 2016. 
[10] R. Wu, W. Peters, and M. W. Morgan, "The next generation of clinical decision support: linking evidence to best practice," Journal of Healthcare Information Management, vol. 16, no. 4, 50 pages, 2002.

[11] U. R. Acharya, O. Faust, N. A. Kadri, J. S. Suri, and W. Yu, "Automated identification of normal and diabetes heart rate signals using nonlinear measures," Computers in Biology and Medicine, vol. 43, no. 10, pp. 1523-1529, 2013.

[12] C. Barbieri, F. Mari, A. Stopper et al., "A new machine learning approach for predicting the response to anemia treatment in a large cohort of end stage renal disease patients undergoing dialysis," Computers in Biology and Medicine, vol. 61, pp. 5661, 2015.

[13] B. Robson and S. Boray, "Implementation of a web based universal exchange and inference language for medicine: sparse data, probabilities and inference in data mining of clinical data repositories," Computers in Biology and Medicine, vol. 66, pp. 82-102, 2015.

[14] S. A. I. Shenas, B. Raahemi, M. H. Tekieh, and C. Kuziemsky, "Identifying high-cost patients using data mining techniques and a small set of non-trivial attributes," Computers in Biology and Medicine, vol. 53, pp. 9-18, 2014.

[15] J. K. Kim, J. S. Lee, D. K. Park, Y. S. Lim, Y. H. Lee, and E. Y. Jung, "Adaptive mining prediction model for content recommendation to coronary heart disease patients," Cluster Computing, vol. 17, no. 3, pp. 881-891, 2014.

[16] L. Verma, S. Srivastava, and P. C. Negi, “A hybrid data mining model to predict coronary artery disease cases using noninvasive clinical data," Journal of Medical Systems, vol. 40, no. 7, pp. 1-7, 2016.

[17] Z. Zhao and C. Ma, "An intelligent system for noninvasive diagnosis of coronary artery disease with EMD-TEO and BP neural network," in 2008 International Workshop on Education Technology and Training \& 2008 International Workshop on Geoscience and Remote Sensing, vol. 2, pp. 631-635, Shanghai, China, 2008.

[18] M. Akay, "Noninvasive diagnosis of coronary artery disease using a neural network algorithm," Biological Cybernetics, vol. 67, no. 4, pp. 361-367, 1992.

[19] M. Kukar, I. Kononenko, C. Grošelj, K. Kralj, and J. Fettich, "Analysing and improving the diagnosis of ischaemic heart disease with machine learning," Artificial Intelligence in Medicine, vol. 16, no. 1, pp. 25-50, 1999.

[20] R. Detrano, A. Janosi, W. Steinbrunn et al., "International application of a new probability algorithm for the diagnosis of coronary artery disease," The American Journal of Cardiology, vol. 64, no. 5, pp. 304-310, 1989.

[21] P. N. Tan, Introduction to Data Mining, Pearson Addison Wesley, San Francisco, 2006.

[22] I. H. Witten, E. Frank, M. A. Hall, and C. J. Pal, Data Mining: Practical Machine Learning Tools and Techniques, Morgan Kaufmann, USA, 2016.

[23] R. Chadha, S. Mayank, A. Vardhan, and T. Pradhan, "Application of data mining techniques on heart disease prediction: a survey," in Emerging Research in Computing, Information, Communication and Applications, pp. 413-426, Springer India, 2016.

[24] H. M. R. Ugalde, J. C. Carmona, J. Reyes-Reyes, V. M. Alvarado, and J. Mantilla, "Computational cost improvement of neural network models in black box nonlinear system identification," Neurocomputing, vol. 166, pp. 96-108, 2015.
[25] A. Shrikumar, P. Greenside, A. Shcherbina, and A. Kundaje, "Not Just a Black box: Learning Important Features through Propagating Activation Differences," 2016, http://arxiv.org/ abs/1605.01713.

[26] D. Sussillo and O. Barak, "Opening the black box: lowdimensional dynamics in high-dimensional recurrent neural networks," Neural Computation, vol. 25, no. 3, pp. 626-649, 2013.

[27] Korea Center for Disease Control and Prevention, The Six Korea National Health \& Nutrition Examination Survey 2013 (KNHANES VI), January 2017, http://knhanes.cdc.go.kr/.

[28] A. G. Mainous, R. J. Koopman, V. A. Diaz, C. J. Everett, P. W. Wilson, and B. C. Tilley, "A coronary heart disease risk score based on patient-reported information," The American Journal of Cardiology, vol. 99, no. 9, pp. 1236-1241, 2007.

[29] S. Capewell, E. S. Ford, J. B. Croft, J. A. Critchley, K. J. Greenlund, and D. R. Labarthe, "Cardiovascular risk factor trends and potential for reducing coronary heart disease mortality in the United States of America," Bulletin of the World Health Organization, vol. 88, no. 2, pp. 120-130, 2010.

[30] A. Field, Discovering statistics using IBM SPSS statstics, Sage, Washington DC, 2013.

[31] J. A. Swets, Signal Detection Theory and ROC Analysis in Psychology and Diagnostics: Collected Papers, Psychology Press, New York, 2014.

[32] S. V. Stehman, "Selecting and interpreting measures of thematic classification accuracy," Remote Sensing of Environment, vol. 62, no. 1, pp. 77-89, 1997.

[33] R. H. Fletcher, S. W. Fletcher, and G. S. Fletcher, Clinical Epidemiology: The Essentials, Lippincott Williams \& Wilkins, USA, 2012.

[34] ISO, Guide to the Expression of Uncertainty in Measurement, International Organization for Standardization, Geneva, Switzerland, 1993.

[35] Z. Arabasadi, R. Alizadehsani, M. Roshanzamir, H. Moosaei, and A. A. Yarifard, "Computer aided decision making for heart disease detection using hybrid neural network-genetic algorithm," Computer Methods and Programs in Biomedicine, vol. 141, pp. 19-26, 2017.

[36] Q. K. Al-Shayea, "Artificial neural networks in medical diagnosis," International Journal of Computer Science Issues, vol. 8, no. 2, pp. 150-154, 2011.

[37] W. G. Baxt, "Application of artificial neural networks to clinical medicine,” The Lancet, vol. 346, no. 8983, pp. 1135-1138, 1995.

[38] D. M. Lloyd-Jones, M. G. Larson, A. Beiser, and D. Levy, "Lifetime risk of developing coronary heart disease," The Lancet, vol. 353, no. 9147, pp. 89-92, 1999.

[39] M. J. Legato, E. Padus, and E. D. Slaughter, "Women's perceptions of their general health, with special reference to their risk of coronary artery disease: results of a national telephone survey," Journal of Women's Health, vol. 6, no. 2, pp. 189198, 1997.

[40] A. Dudina, M. T. Cooney, D. D. Bacquer et al., "Relationships between body mass index, cardiovascular mortality, and risk factors: a report from the SCORE investigators," European Journal of Cardiovascular Prevention \& Rehabilitation, vol. 18, no. 5, pp. 731-742, 2011.

[41] M. Ezzati, S. Vander Hoorn, A. Rodgers et al., "Estimates of global and regional potential health gains from reducing multiple major risk factors," The Lancet, vol. 362, no. 9380, pp. 271-280, 2003. 
[42] G. Assmann, H. Schulte, A. von Eckardstein, and Y. Huang, "High-density lipoprotein cholesterol as a predictor of coronary heart disease risk. The PROCAM experience and pathophysiological implications for reverse cholesterol transport," Atherosclerosis, vol. 124, pp. S11-S20, 1996.

[43] S. MacMahon, R. Peto, R. Collins, J. Godwin, J. Cutler, P. Sorlie et al., "Blood pressure, stroke, and coronary heart disease: part 1, prolonged differences in blood pressure: prospective observational studies corrected for the regression dilution bias," The Lancet, vol. 335, no. 8692, pp. 765-774, 1990.

[44] W. B. Kannel, "Blood pressure as a cardiovascular risk factor: prevention and treatment," JAMA, vol. 275, no. 20, pp. 1571-1576, 1996.

[45] W. C. Willett, A. Green, M. J. Stampfer et al., "Relative and absolute excess risks of coronary heart disease among women who smoke cigarettes," New England Journal of Medicine, vol. 317, no. 21, pp. 1303-1309, 1987.

[46] E. L. Barrett-Connor, B. A. Cohn, D. L. Wingard, and S. L. Edelstein, "Why is diabetes mellitus a stronger risk factor for fatal ischemic heart disease in women than in men? The Rancho Bernardo Study," JAMA, vol. 265, no. 5, pp. 627-631, 1991.

[47] R. C. Sole, C. T. Lucas, L. U. Rivera, D. C. Salazar, and M. A. Saldaña, "[pp. 29.15] Obesity increases the central systolic and diastolic blood pressure despite having proper treatment in hypertensive patients," Journal of Hypertension, vol. 34, article e303, 2016.

[48] Y. Aoki, S. S. Yoon, Y. Chong, and M. D. Carroll, "Hypertension, abnormal cholesterol, and high body mass index among non-Hispanic Asian adults: United States, 2011-2012," NCHS Data Brief, vol. 140, pp. 1-8, 2014. 


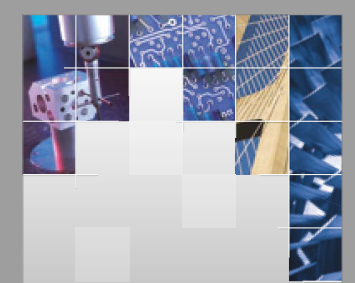

\section{Enfincering}
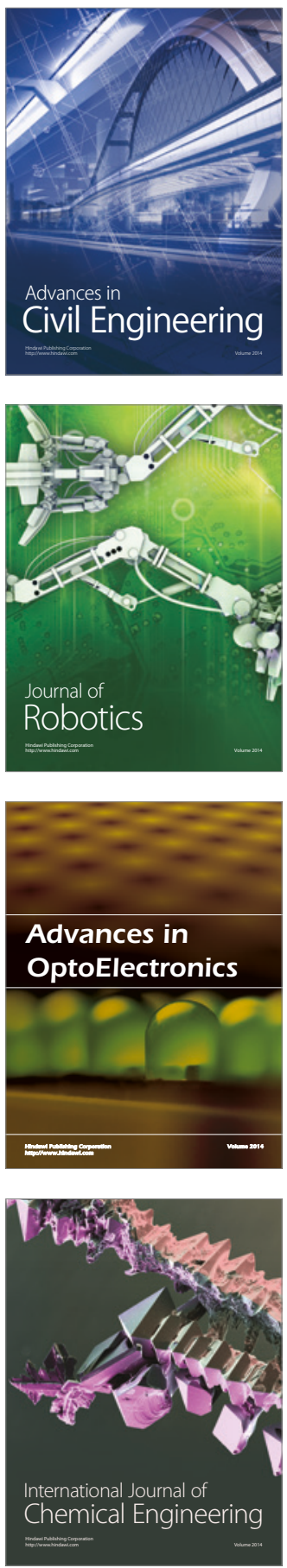

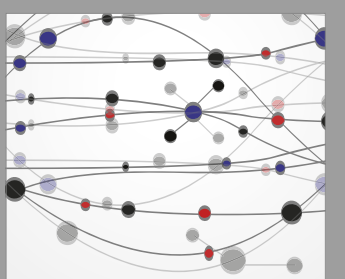

The Scientific World Journal

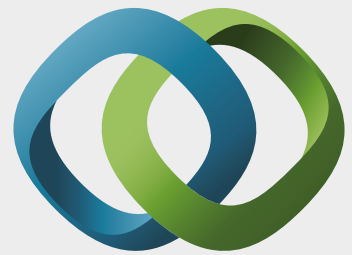

\section{Hindawi}

Submit your manuscripts at

https://www.hindawi.com
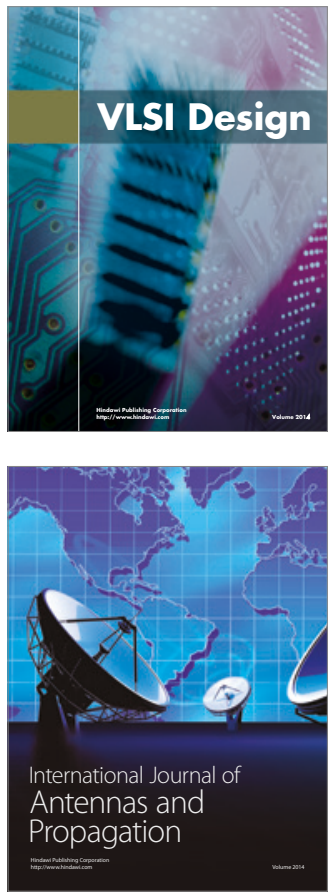

\section{Rotating}

Machinery
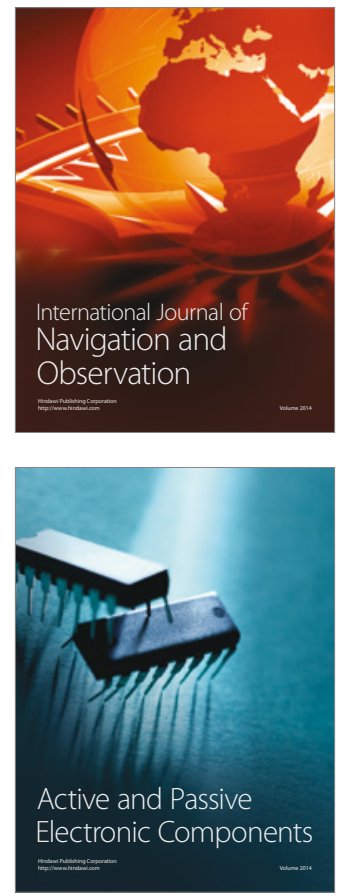
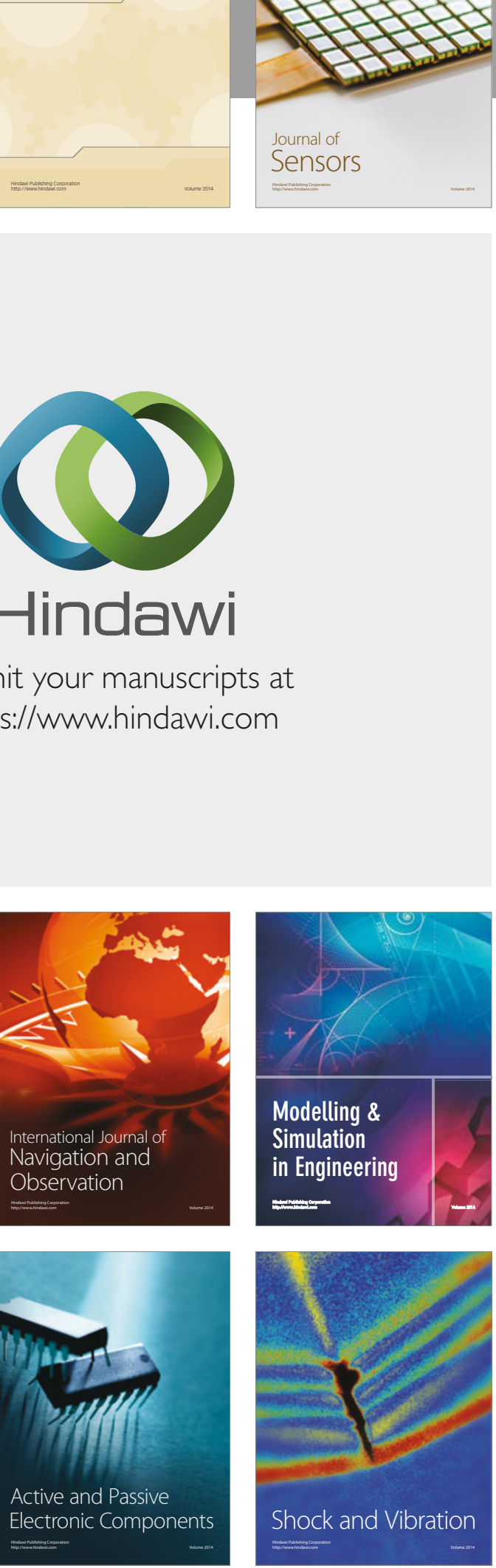
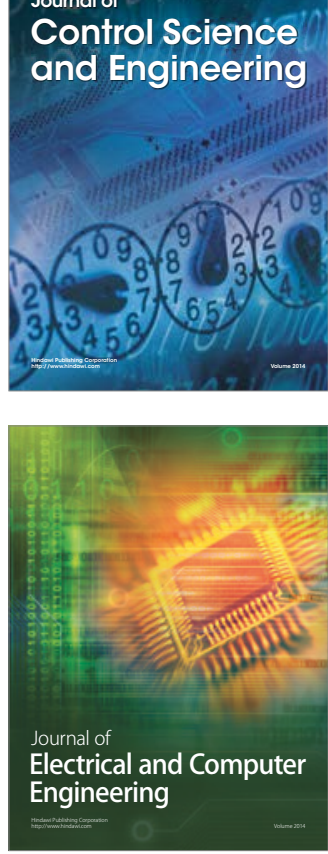

Distributed

Journal of

Control Science

and Engineering
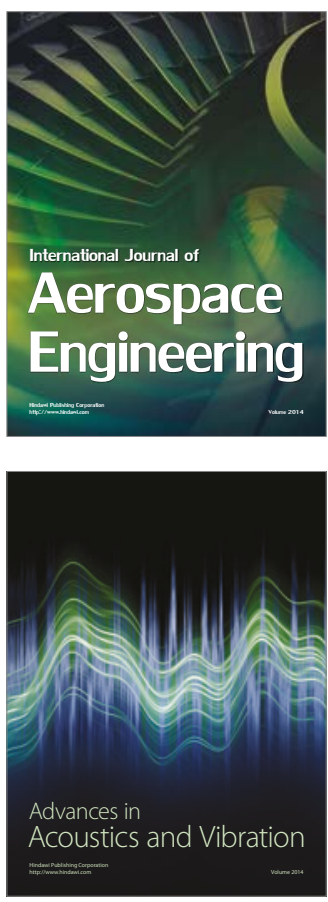

Sensor Networks 